\title{
Effect of General Anesthesia on Levels of Blood Sugar Values in Different Times of Operation
}

\author{
Anaam Khamis Abd ${ }^{1}$, Ghadeer Hussein Taha', \\ Qater Al-nada Kanaem Ali Al-Ibady ${ }^{3}$, Hussam Kareem Mughamis ${ }^{4}$ \\ ${ }^{1}$ Student, ${ }^{2}$ Lecturer, ${ }^{3}$ Assist. Prof., 3Anesthetist, Department of Anesthesia, College of Health and Medical Technics, \\ Middle Technical University, Baghdad, Iraq, ${ }^{4}$ MB.Ch.BlFICMS Anesth and IC, Al-Imam Ali General Hospital
}

\begin{abstract}
The present study was carried out on a period between December 2019 to February 2020 on 50 healthy patients, their ages ranged from eighteen to fifty years. They were 26 males and 24 females. These healthy patients were attending Baghdad Educational Hospital, Al-Harriri Hospital, and Al-Diwaniyah General Hospital in order to perform various surgeries. And after all tests are done. Then they were admitted to the operating theater. In this study used the general anesthesia method. And they were given drugs of anesthesia, during each step; the values of blood sugar levels $(\mathrm{Mg} \backslash \mathrm{dl})$ were measured. Such as pre induction, post induction, after incision, after fluid, and recovery. To know the effect of general anesthesia method on blood sugar levels in different times of operation on gender and age groups.

This study showed that total numbers of patients were 50 including males about $26(52.0 \%)$ and females about 24(48.0\%), their age of all individuals ranged from less than 20 years old $(<20-29)$ which number and percentage $22,44.0 \%$ to 50 years old $7(14.0 \%)$. Also there are no significant difference was observed in the blood sugar levels changes (Mgldl) in both males and females (gender) before and after the operation or surgery, also no found any differences between blood sugar levels changes ( $\mathrm{Mg} \backslash \mathrm{dl})$ at the age groups and the general anesthesia method, after and before the operation in different times of surgery. So in this study summarized the mean and stander deviation values according to the levels of blood sugar (Mgldl) among different times of surgery, and then show the mean of pre induction period about 83.84, While mean of values of blood sugar at post induction time at general anesthesia method about 104.94.
\end{abstract}

Aims and Objectives: This study was investigated the influence of general anesthetic drugs that effect on the blood sugar level changes of patients with surgery. This was done with the following objectives:

- To administer general anesthesia by using a different combination of drugs.

- To assess and compare the blood sugar level changes of the patients at different time intervals.

- Complications, if any were observed and noted on patients at different time intervals.

Keywords: General Anesthesia; Blood Sugar; Times of Operation.

\section{Introduction}

Anesthesia was not known prior to 1846 although the anaesthetic properties of ether already described by Faraday in 1818 .the use of ether in 1846 by W.T.G mortan opened up anew era of painless surgery with the help of drugs.Presently there are various anaesthetic agents ether still stand out as the most commonly used anesthetic agent in our country ${ }^{[1]}$. the primary goal of anesthesia is to provide patient comfortt and safety during surgery or to facilitate the surgical procedure itself. Anesthesia can be general, regional, and local, depending on the needs of the patient and surgeon. General anesthesia is a reversible drug induced state of unconsciousness with hypnosis, amnesia,analgesia and elimination of the patient response to the painful stimuli sometimes accompanied by paralysis ${ }^{[2]}$.

Glucose the main conversion product of carbohydrate food enters the blood from the intestine. Glucose is distributed fairly uniformly throughout the body fluid both extracellular and intracellular. Maintenance of 
blood sugar at a constant level is a balance between production and loss ${ }^{[1]}$. Blood glucose concentrations in normal healthy individuals are normally maintained at $\sim 90 \mathrm{mg} / \mathrm{dl}$.Abnormal concentrations of glucose in plasma result in deleterious effects at the whole organism level. Glucose is the main energy source for the brain and decreased plasma glucose levels (hypoglycemia) can lead to impaired brain function and death. Conversely, increased plasma glucose levels (hyperglycemia), a major clinical symptom of diabetes ${ }^{[3]}$. The key hormones which regulate glucosehomoeostasis include insulin, glucagon, epinephrine, norepinephrine, cortisol and growth hormone $(\mathrm{GH})^{[4]}$. Surgeries are considered to be the combination of multiple factors including tissue damage, fasting, blood-loss, effect of medication and temperature changes from a metabolic point of view. Combinations of all these factors give rise to stress response. The stress response to surgery is characterized by increased secretion of pituitary hormones and activation of the sympathetic nervous system. The ultimate effect of these various endocrine changes is increased catabolic activity by increased secretion of catabolic hormones like cortisol and glucagon. The effect of these endocrine and metabolic changes ultimately leads to increased neoglucogenesis and hyperglycemia. So this stress response may be quantified by the incidence of hyperglycemia.

The metabolic changes appear to be proportional to the severity of the surgical trauma with plasma cortisol and blood glucose concentration rising slightly during minor surgical procedures but significantly during major operations ${ }^{[5]}$. Regulation blood sugarlevels in the use of general anesthesia needs to be considered carefully.Some induction agents for general anesthesia havetheside effects of increasing blood sugar level ${ }^{[6]}$. Anesthetic drugs will basically have a sympathomimetic effect and affect the endocrine system in the human body, especially the regulation of blood glucose levels. The mechanism for increasing blood glucose levels is very complex. One opinion held is that anesthetic drugs directly suppress pancreatic beta cells through the release of catecholamines and result in decreased insulin production $^{[7]}$. All the intravenous agents and volatile anesthetics in normal doses have minor influence on the endocrine and metabolic function. Severity of surgery and the type of anesthesia influence the magnitude of the counter regulatory response which is evidenced byincrease in circulating catecholamines, cortisol and glucagon concentration and blood glucose aswell[ ${ }^{[5]}$.

\section{Materials and Method}

The current study was done in Baghdad Educational Hospital, Al-Harriri Hospital and Al-Diwaniyah General Hospital during the year 2019-2020,with the aim to study effect of general anesthesia on blood sugar level in patients undergoing surgery. The study recruited 50 adult patient, all the patients were of physically fit belonging to A.S.A. grade I or II,between the age of 18 to 50 years who were ASAI and ASAII patients of both sexes presenting for elective surgeries.

Anesthetic Technique: In the operating room, the standard anesthetic machine check was done prior to commencement of any procedure every morning. All patients had baseline blood glucose measurement performed immediately prior to the start of any things. The patients were cannulated using 18 or 20 or $22 \mathrm{G}$ cannula and an infusion of normal saline or normal saline with glucose was initiated. Non invasive blood pressure cuff, ECG and pulse oximeter probe were then connected to the patient and initial blood pressure and heart rate readings were then obtained. All patients were premedicated with $1 \mathrm{mg}$ midazolam I.V, ranitidine $50 \mathrm{mg}$ I.V, fentanyl $2 \mathrm{mcg} / \mathrm{kg} \mathrm{I.V}$, metclopromide $10 \mathrm{mg}$ I.V and dexamethasone $8 \mathrm{mg}$ I.V,3 minutes prior to induction. Pre-oxygenation was done during these three minutes after which, induction of anaesthesia using I.V. propofol 2-3 $\mathrm{mg} / \mathrm{kg}$, I.V.ketamine $1-2 \mathrm{mg} / \mathrm{kg}$ was done.For the ETT, muscle relaxation for intubation was facilitated by the use of injection rocuronium $0.6 \mathrm{mg} / \mathrm{kg}$ or atracurium $0.5 \mathrm{mg} / \mathrm{kg}$.

Patients were then ventilated with 100 percent oxygen for a period of 1 minute prior to intubation with the aid of Macintosh laryngoscope. Endotracheal tubes of size $7 / 7.5$ for female and $8 / 8.5$ for male patients were used depending on body weight.Anaesthesia was maintained using oxygen and sevoflurane or isofluraneAdequacy of ventilation was monitored clinically by assessing chest expansion, auscultation of the lung fields and the epigastric region. Surgeryorany other manipulations were not allowedtocommencetillthe study was completed i.e. for ten minutes afterintubation/ insertion.

Before end of the operation in a short time, the patient is given paracetamol $1000 \mathrm{mg}$ to relieve pain after the operation.

Study Tools: During the operatation, blood sugar is measured 5 times for the patient by using electronic 
blood glucose meter, the first time is measured preinduction of anesthesia, the second time is measured post induction of anesthesia, the third time after surgeon begin the operation, the fourth time after stop the fluid and the fifth time is measured in the recovery room. Method of measurement by applying a drop of blood to a chemically treated, disposable 'test-strip', which is then inserted into an electronic blood glucose meter. The reaction between the test strip and the blood is detected by the meter and displayed in units of $\mathrm{mg} / \mathrm{dL}$ or $\mathrm{mmol} / \mathrm{L}$.

\section{Results}

The demographical picture of studied groups in table 1 , for this study showed that total numbers of patients were 50 including males about $26(52.0 \%)$ and females about $24(48.0 \%)$, their age of all individuals ranged from less than 20 years old $(<20-29)$ which number and percentage $(22,44.0 \%)$ to 50 years old $(7,14.0 \%)$. There are no significant difference was observed in the blood sugar levels changes of both males and females (gender) before and after the operation or surgery, also no found any differences (not a statistically significant difference) between blood sugar levels at the age groups and the general anesthesia method, after the operation in different times of surgery.

\section{Table (1): Distribution of group study according to the gender and age groups (year)}

\begin{tabular}{|l|l|c|c|}
\hline \multicolumn{2}{|l|}{ The Cases (Patients) } & No.(n=50) & $\begin{array}{c}\text { Percentage } \\
(\mathbf{1 0 0 \%})\end{array}$ \\
\hline \multirow{3}{*}{ Gender } & Male & 26 & 52.0 \\
\cline { 2 - 4 } & Female & 24 & 48.0 \\
\hline \multirow{3}{*}{$\begin{array}{l}\text { Age groups } \\
\text { (year) }\end{array}$} & $(<20-29)$ & 22 & 44.0 \\
\cline { 2 - 4 } & $(30-49)$ & 21 & 42.0 \\
\cline { 2 - 4 } & $(+50)$ & 7 & 14.0 \\
\hline
\end{tabular}

But the results in table 2, summarized the mean and stander deviation values according to the levels of blood sugar $(\mathrm{Mg} \backslash \mathrm{dl})$ among different times of surgery, that show the mean of pre induction period about 83.84, but the stander deviation for this period about 13.610. While the mean of values blood sugar at post induction time at general anesthesia method about 104.94. Also in this table show increase the values of blood sugar in other periods at general anesthesia method such as after incision, and after fluid, that was about 128.66, 137.56 respectively, but in recovery time the mean of value of blood sugar reached to about 125.94.
Table (2): Mean and Std. Deviation values of Blood sugar (Mg\dl) among different time

\begin{tabular}{|l|c|c|}
\hline The Times & Mean & Std. Deviation \\
\hline Pre induction & 83.84 & 13.610 \\
\hline Post induction & 104.94 & 35.720 \\
\hline After incision & 128.66 & 74.893 \\
\hline After fluid & 137.56 & 33.739 \\
\hline Recovery & 125.94 & 29.398 \\
\hline
\end{tabular}

Then the table 3, shows the compare between values levels blood sugar ( $\mathrm{Mg} \backslash \mathrm{dl})$ changes at pre inductionand other times. This table show great differences between the two times that were pre induction and post induction. This means found highly significant between the two means values blood sugar ( $\mathrm{Mg} \backslash \mathrm{dl}$ ) atpre inductionandpost induction for general anesthesia method. So above applies topre induction and other times atgeneral anesthesia method under probability level $(\mathrm{P}<0.01)$.

\section{Table (3): Compare between values of Blood sugar} $(\mathrm{Mg} \backslash \mathrm{dl})$ at Pre induction and other times

\begin{tabular}{|l|c|c|c|}
\hline The parameters of times & t-test & P-Value & C.S \\
\hline Pre induction - Post induction & 4.564 & .000 & $\begin{array}{c}\mathrm{P}<0.01 \\
(\mathrm{HS})\end{array}$ \\
\hline Pre induction - After incision & 4.208 & .000 & $\begin{array}{c}\mathrm{P}<0.01 \\
(\mathrm{HS})\end{array}$ \\
\hline Pre induction - After fluid & 10.354 & .000 & $\begin{array}{c}\mathrm{P}<0.01 \\
(\mathrm{HS})\end{array}$ \\
\hline Pre induction - Recovery & 9.557 & .000 & $\begin{array}{c}\mathrm{P}<0.01 \\
(\mathrm{HS})\end{array}$ \\
\hline
\end{tabular}

While thetable 4, show the compare between values of blood sugar $(\mathrm{Mg} \backslash \mathrm{dl})$ at Post induction and other times. This table show great differences between the two times that were post induction andafter incision. This means found highly significant between the two means values blood sugar $(\mathrm{Mg} \backslash \mathrm{dl})$ atpost inductionandafter incision for general anesthesia method. And above applies topost induction and other times atgeneral anesthesia method under probability level $(\mathrm{P}<0.01)$.

\section{Table (4): Compare between values of Blood sugar (Mg\dl) at Post induction and other times}

\begin{tabular}{|l|c|c|c|}
\hline The parameters of times & t-test & P-Value & C.S \\
\hline Post induction-After incision & 2.123 & .039 & $\mathrm{P}<0.01(\mathrm{HS})$ \\
\hline Post induction-After fluid & 5.033 & .000 & $\mathrm{P}<0.01(\mathrm{HS})$ \\
\hline Post induction-Recovery & 3.365 & .001 & $\mathrm{P}<0.01(\mathrm{HS})$ \\
\hline
\end{tabular}


Also the table 5, demonstrated compare between the values of blood sugar levels $(\mathrm{Mg} \backslash \mathrm{dl})$ at after incision and other times. So this table shows no found differences between the two times which wereafter incision and after fluid. And this means no found significant differences between two means values blood sugar $(\mathrm{Mg} \backslash \mathrm{dl})$ atafter incision andafter fluid for general anesthesia method. And above applies tothe period after incision and recovery atgeneral anesthesia method under probability level $(\mathrm{P}<0.01)$.

\section{Table (5): Compare between values of Blood sugar (Mg\dI) at after incision and other times}

\begin{tabular}{|l|c|c|c|}
\hline The Parameters of times & t-test & P-Value & C.S \\
\hline After incision - After fluid & 0.831 & .410 & $\mathrm{P}>0.05$ (NS) \\
\hline After incision - Recovery & 0.259 & .797 & $\mathrm{P}>0.05$ (NS) \\
\hline
\end{tabular}

Finally the Table 6 , show that compare between values of blood sugar levels ( $\mathrm{Mg} \backslash \mathrm{dl})$ at after fluid and recovery. Also this result in this table shows differences between the two periods that wereafter fluid and recovery. While this result means found significant differences between two means values blood sugar $(\mathrm{Mg} \backslash$ dl) atafter fluid andrecovery for this general anesthesia method under probability level $(\mathrm{P}<0.01)$.

Table (6): Compare between values of Blood sugar (Mg\dl) at after fluid - Recovery

\begin{tabular}{|l|c|c|c|}
\hline The Parameters of times & t-test & P-Value & C.S \\
\hline After fluid - Recovery & 2.075 & .043 & $\mathrm{P}<0.05$ (S) \\
\hline
\end{tabular}

\section{Discussion}

In this study, there are no significant difference was observed in the blood sugar levelschanges in gender before and after the operation or surgery, also no found any differences (not a statistically significant differences) between blood sugar levels changes at the age groups and the general anesthesia method, after the operation in different times of surgery. Therefore this result is consistent with research of Kumar et al ${ }^{[8] \text {, }}$ when they also found the bloodglucose measurements at various time intervals were compared between two groups, while no significant statistical difference was found preoperatively and after induction of anesthesia, significant statistical difference in blood sugar level changes was found $1 / 2$ after induction of anesthesia, at the end of anesthesia and $1 \mathrm{~h}$ after end of anesthesia.
From the present study we concluded that in comparison of muscle relaxant ether causes much more hyperglycemia during general anesthesia which is very highly significant rise. But in case of muscle relaxant the change in blood glucose level is just significant. There are because the glucose conceder as the main conversion product of carbohydrate food enters the blood from the intestine. Glucose is distributed fairly uniformly throughout the body fluid both extracellular and intracellular. Maintenance of the blood sugar at a constant level is a balance between production and $\operatorname{loss}^{[9]}$. While this result dis agree with Kouzegaran et $a l^{[10]}$, when reached to the patients had no taken or were not diagnosed with any diseases affecting blood sugar levels. They found no significant difference observed in the blood sugar levels of both groups before and after the operation, but the group which had utilized the general anesthesia method reported with lower levels of blood sugar than the spinal anesthesia group after operation with a significant difference $(\mathrm{p}<0.05)$. A difference has been observed in the levels of blood sugar 2, 12 and 24 hours after the operation in both groups, but it was not a statistically significant difference. As the results indicate, both the general and spinal anesthesia are effective method to reduce the serum level of blood sugar in patients undergoing caesarean. However, none of mentioned method had any influence on reduction of blood sugar levels during the recovery or the period after it. However, none of the mentioned method had any influence on reduction of blood sugar levels during the recovery or the period after it. While the compare between values levels blood sugar ( $\mathrm{Mg} \backslash \mathrm{dl})$ at pre inductionand other times. There are great differences between the two times that were pre induction and post induction. This result agree with the study of Maitra et al., [11], that was undertaken to observe the effect of different maintenancefluid regimen on intraoperative blood glucose levels in non-diabetic patients undergoing elective major noncardiac surgery under general anesthesia. That stress induced-hyperglycemic response in patients undergoing major non-cardiac surgery is common in non-diabetic population. Maintenance-fluid therapy by dextrose containing solution as opposed to Ringer's lactate solution increases the incidence of hyperglycemia. To achieve normoglycemia by intravenous bolus dose of human regular insulin, significantly higher doses are required in patients receiving dextrose containing saline as maintenance fluid. However, they did not mention their intraoperative fluid protocol. Both Saringcarinkul and Kotrawera [12], found a progressive increase in the 
blood glucose values in patients receiving 5\% dextrose during surgery.

\section{Conclusion}

1. The levels of blood sugar ( $\mathrm{Mg} \backslash \mathrm{dl})$ changes among different times of surgery ingeneral anesthesia method, that show increase the values of blood sugar in other periods at general anesthesia method such as after incision, and after fluid.

2. Found highly significant differences between means values blood sugar $(\mathrm{Mg} \backslash \mathrm{dl})$ at pre inductionandpost induction and between the pre induction - after incision, pre induction - after fluid, and pre induction - recovery for general anesthesia method. Also found highly significant differences between the two means values blood sugar $(\mathrm{Mg} \backslash \mathrm{dl})$ at post inductionandafter incision for general anesthesia method. These because increase the stress hormone, nervous tension, and psychological tension, fear, and terror during the surgery.soFound significant differences between two means values blood sugar $(\mathrm{Mg} \backslash \mathrm{dl})$ at after fluid andrecovery for this general anesthesia method. Because the fear and terror from the idea of performing the operation. To increase the stress hormone specialized in stress factors on the body, which makes the body alert attention to what happens to him whenever occur a surgical incision.

\section{Conflict of Interest: None}

Source of Findings: None

Ethical Clearance: None

\section{References}

1. Krishna Reddy, G.V., M. Madan Mohan Rao, G obulesu, R Salma Mahaboob. The study of blood glucose level changes during general anesthesia in patients undergoing surgery. IAIM, 2017; 4(1): 110-115.

2. Vacanti, Charles, et al., eds. Essential clinical anesthesia. Cambridge University Press, 2011.

3. Sharabi, Kfir, et al. "Molecular pathophysiology of hepatic glucose production." Molecular aspects of medicine 46 (2015): 21-33.

4. Mitrakou, Asimina. "Kidney: its impact on glucose homeostasis and hormonal regulation." Diabetes research and clinical practice 93 (2011): S66-S72.

5. Chakrabarti, S., S. D. Gupta, and I. Chakraborty. "Intraoperative blood glucose level changes between obese and non-obese non-diabetic patients undergoing general anaesthesia for creniotomy surgeries." J of Anes and Cri Open Access 10.5 (2018): 162-165.

6. Brown, Emery N., Ralph Lydic, and Nicholas D. Schiff. "General anesthesia, sleep, and coma." New England Journal of Medicine 363.27 (2010): 26382650.

7. Kumar, Manoj, et al. "Influence of two anesthetic techniques on blood sugar level in head injury patients: a comparative study." Anesthesia, essays and researches 10.2 (2016): 207.

8. Kumar, Manoj, et al. "Influence of two anesthetic techniques on blood sugar level in head injury patients: a comparative study." Anesthesia, essays and researches 10.2 (2016): 207.

9. Furnary, Anthony P., et al. "Continuous insulin infusion reduces mortality in patients with diabetes undergoing coronary artery bypass grafting." The Journal of thoracic and cardiovascular surgery 125.5 (2003): 1007-1021.

10. Kouzegaran, Samaneh, Hoda Sarjughi, and Amir Saber Tanha. «Comparing the effects of general anesthesia and spinal anesthesia on the serum level of blood sugar in patients undergoing cesarean.» Interventional Medicine and Applied Science 10.4 (2018): 202-206.

11. Maitra, Souvik, et al. "Intraoperative blood glucose levels in nondiabetic patients undergoing elective major surgery under general anaesthesia receiving different crystalloid solutions for maintenance fluid." Anesthesia, essays and researches 7.2 (2013): 183.

12. Saringcarinkul, Ananchanok, and Kriengsak Kotrawera. «Plasma glucose level in elective surgical patients administered with 5\% dextrose in $0.45 \% \mathrm{NaCl}$ in comparison with those receiving lactated Ringer>s solution.» Medical journal of the Medical Association of Thailand 92.9 (2009): 1178 . 